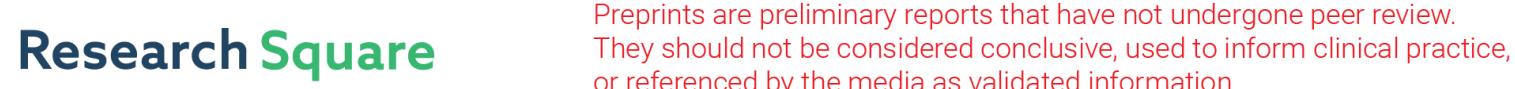 or referenced by the media as validated information. \\ Invasion front dynamics of interactive populations in environments with barriers
}

\section{Youness Azimzade ( $\square$ younessazimzade@gmail.com )}

University of Tehran

\section{Research Article}

Keywords: growth exponent, invasion, cancer cells, Langevin equation, environmental stress

Posted Date: July 26th, 2021

DOl: https://doi.org/10.21203/rs.3.rs-738579/v1

License: (c) (i) This work is licensed under a Creative Commons Attribution 4.0 International License. Read Full License

Version of Record: A version of this preprint was published at Scientific Reports on January 17th, 2022. See the published version at https://doi.org/10.1038/s41598-022-04806-x. 


\title{
Invasion front dynamics of interactive populations in environments with barriers
}

3 Youness Azimzade ${ }^{1, *}$

\author{
${ }^{1}$ Department of Physics, University of Tehran, Tehran 14395-547, Iran \\ *younessazimzade@gmail.com
}

6 ABSTRACT

Invading populations normally comprise different subpopulations that interact while trying to overcome existing barriers against their way to occupy new areas. However, the majority of studies so far only consider single or multiple population invasion into areas where there is no resistance against the invasion. Here, we developed a model to study how cooperative/competitive populations invade in the presence of a physical barrier that should be degraded during the invasion. For one dimensional environment, we found that a Langevin equation as $d X / d t=V_{f} t+\sqrt{D_{f}} \eta(t)$ describes invasion front position. We then obtained how $V_{f}$ and $D_{f}$ depend on population interactions and environmental barrier intensity. For the 2D case, for the average interface position we found a Langevin equation as $d H / d t=V_{H} t+\sqrt{D_{H}} \eta(t)$. Similar to the $1 \mathrm{D}$ case, we found how $V_{H}$ and $D_{H}$ respond to population interaction and environmental barrier intensity. Finally, the study of invasion front morphology through dynamic scaling analysis showed that growth exponent, $\beta$, depends on both population interaction and environmental barrier intensity. Saturated interface width, $W_{\text {sat }}$, versus width of the 2D environment $(L)$ also exhibits scaling behavior. Comparing results for the 2D environment revealed that competition among subpopulations leads to more rough invasion fronts. Considering the wide range of shreds of evidence for clonal diversity in cancer cell populations, our findings suggest that interactions between such diverse populations can potentially participate in the geometry of the tumor border.

\section{Introduction}

Invasion is a generic process that emerges across different scales and populations ${ }^{1,2}$. During the invasion, new, possibly fitter species occupy further areas mostly at the expense of extinction existing populations, putting the existing populations in danger. As such, understanding how invasion happens and what related parameters regulate it is of great interest across different fields ${ }^{3-5}$. The invasion has been under investigation for about a century by mathematicians and physicists ${ }^{6}$. Yet, many questions remained to be tackled, particularly where invasion and evolutionary processes are interrelated ${ }^{7}$.

When tumor cells invade into surrounding tissues, invasion becomes a concerning health threat. Thus, understanding the tumor invasion is not only of theoretical interest, but it also can reveal driving mechanisms behind aggressive behavior ${ }^{8}$. In invasive tumors, cancer cells take over the host tissue by pushing existing healthy cells ${ }^{9}$ and degrading physical structure extracellular matrix (ECM) ${ }^{10-12}$ alongside various chemical and mechanical interactions ${ }^{13,14}$. Facing such a barrier can affect the evolutionary dynamics of tumors in different aspects ${ }^{15}, 16$. More importantly, tumor cells that push the healthy tissue belong to different clones $^{17}$. These subpopulations may cooperate ${ }^{18-21}$ or compete ${ }^{22-24}$ with each other during their way to invade the surrounding healthy tissue ${ }^{25}$. Despite huge literature on clonal diversity in tumors, it is not clear that how such interactions regulate invasion and how the intensity of environmental barriers restricts invasion.

For invasion that emerges as a result of consecutive duplication and migration of species, one can write ${ }^{6,26} \dot{C}=R(C) C+$ $\nabla(D \nabla C)$ where $C, R$ and $D$ represent population density, duplication rate and diffusion constant, respectively. Such a model predicts that invasion happens through traveling waves with velocity of $V_{f}=2 \sqrt{R D}$. Adding number fluctuations to this model leads to fluctuations in propagating waves. For most cases, a Langevin equation provides appropriate representation for these invasion fronts. For such a case, for invasion front, $X$, one can write $d X / d t=V_{f} t+\sqrt{D_{f}} \eta(t)$ where $\eta$ is noise and $\left\langle\eta(t) \eta\left(t^{\prime}\right)\right\rangle=\delta\left(t-t^{\prime}\right)^{27-29}$. Such analysis suggests that by finding $V_{f}$ and $D_{f}$ one can describe invasion at least $1 \mathrm{D}$. In two or higher dimensions, invasion fronts can exhibit additional features such as roughness that can provide additional information too $^{30,31}$.

The geometry of tumor cells' invasion front has been studied from different perspectives. Part of this interest originated from the observation that the geometry of the invasion front is associated with tumor outcome ${ }^{32,33}$. On the other hand, the notion that invasion front geometry might reveal the driving mechanism behind the invasion ${ }^{34,35}$ has sparked various studies on scaling properties of cancer cells invasion front in vivo ${ }^{36-38}$ and using different mathematical models ${ }^{39-43}$. Despite the development of a diverse range of models on tumor invasion, clonal interaction remained overlooked.

Motivated by interactions for cancer cells and inspired by a model on cooperative populations in the presence of environ- 
mental barriers ${ }^{44}$, we developed a model to study how environmental stress regulates invasion front of interactive species. For the 1D case, we tried to see whether any Langevin equation, as predicted by stochastic reaction-diffusion studies, described invasion front movements and then obtained corresponding dependencies on environmental stress for cooperative/competitive populations. For the 2D environment, after finding the Langevin equation of invasion front, we considered it a growing interface and studied how scaling exponents depend on environmental stress and interspecific interactions.

\section{Model}
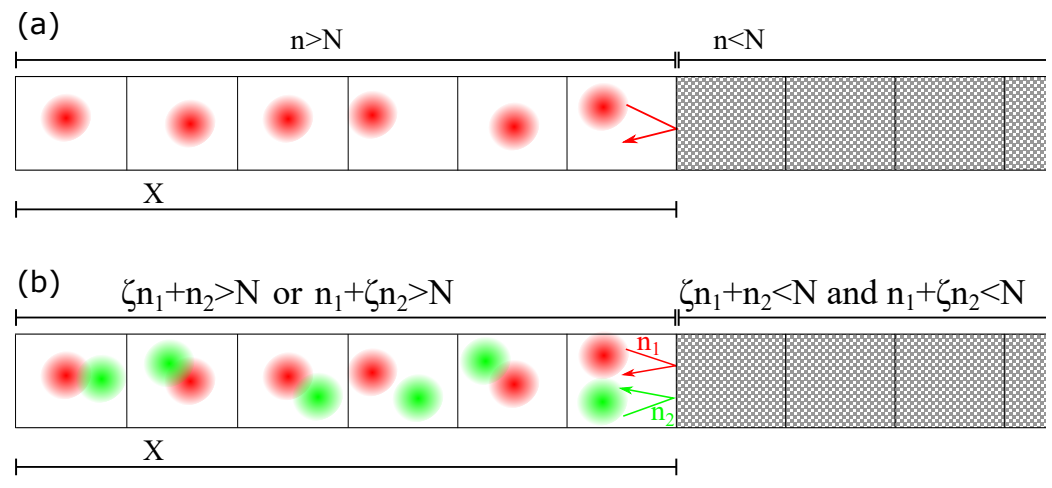

Figure 1. (a) Schematic illustration of the single-species model in one dimension. The red arrow shows a failed trial to occupy a empty nearest neighbor. While this attempt has failed, the strength of the barrier has decreased by 1 . In simple single-species model, after $n=N$ trials, the unit becomes occupiable. (b) Schematic illustration of the two-species model in one dimension. In a randomly selected unit, each species tries to occupy an empty nearest neighbor. We should have $\zeta n_{1}+n_{2}>N$ or $n_{1}+\zeta n_{2}>N$ for a unit to become occupiable.

The single-species model in a 1D environment follows these rules: A unit will be selected randomly. If the unit does contain a species, then $i$ ) it decides to duplicate into one of their empty nearest neighbors (NNs) and would do so if that unit has already been visited and is occupiable. If the selected $\mathrm{NN}$ has not been visited and is not occupiable, the trial number for that $\mathrm{NN}, n$, increases by one (barrier intensity decreases by one). ii) Independent of the duplication process, the species migrate to an NN and do so if the selected $\mathrm{NN}$ is occupiable. If the selected $\mathrm{NN}$ is not occupiable, the trial number for that unit, $n$, increases by one (barrier intensity decreases by one). iii) If migration happens, that unit would be called visited and any unit would be occupiable after $n \geq N$ times being selected for migration or duplication where $N$ is environmental barrier intensity (see Figure 1 (a)).

In the two-species model, for simplicity, we consider species to be able to occupy a unit simultaneously. Due to this assumption, they do not compete for space and interaction between species is limited to their mutual try to degrade the environmental barrier at invasion front ${ }^{44}$. In this case, each species in a randomly selected unit evolves independently based on the aforementioned rules (i-iii) and we count their trials separately as $n_{1}$ and $n_{2}$. For an entirely cooperative scenario, if the number of trials on a unit together exceeds the barrier intensity, $n_{1}+n_{2}>N$, the unit becomes occupiable. In a more complex scenario, a unit would be occupiable if we have $\zeta n_{1}+n_{2}>N$ or $n_{1}+\zeta n_{2}>N$ in which $\zeta$ is the interaction parameter. When two populations are cooperative (competitive) we have $\zeta>0(\zeta<0)$. We anticipate a Langevin equation for invasion front, $X$ (see Figure 1 (b)), and we would try to find out how the diffusion constant and velocity of this interface is related to environmental barrier intensity and interspecific interactions.

\section{Results}

\subsection{D Case}

First, we study the one-dimensional case. We locate a cell at the first unit of a half limited array and let the system evolve based on the above-mentioned rules. We call the occupied unit with the largest distance from the origin as the invasion front (border) location and call its index as $X$. To find the behavior of invasion front and quantify it, we analyze $X, \bar{X}$ and $X-\bar{X}$. Analysis of $X-\bar{X}$ versus time (Figures 2 (a) and (b)) shows that while $N$ affects the magnitude of fluctuations for $X-\bar{X}$, the mean squared displacement behaves like a simple random walk and we have: $\left\langle(X-\bar{X})^{2}\right\rangle \sim t$. As a result, we can define a diffusion constant for these fluctuations as $\left\langle(X-\bar{X})^{2}\right\rangle=D_{f} t$. Then we studied the dependency of $D_{f}$ on $N$. It appeared that for large values of $N$ we have $D_{f} \propto N^{-\gamma_{D}}$ with $\gamma_{D}=2.00 \pm 0.05$ (Figure2 (c)). The averaged velocity of the front position gives us the invasion velocity, $V_{f}$. Invasion velocity also depends on $N$ as $V_{f} \propto N^{-\gamma_{V}}$ with $\gamma_{V}=1.00 \pm 0.05$ (Figure2 (c)). 

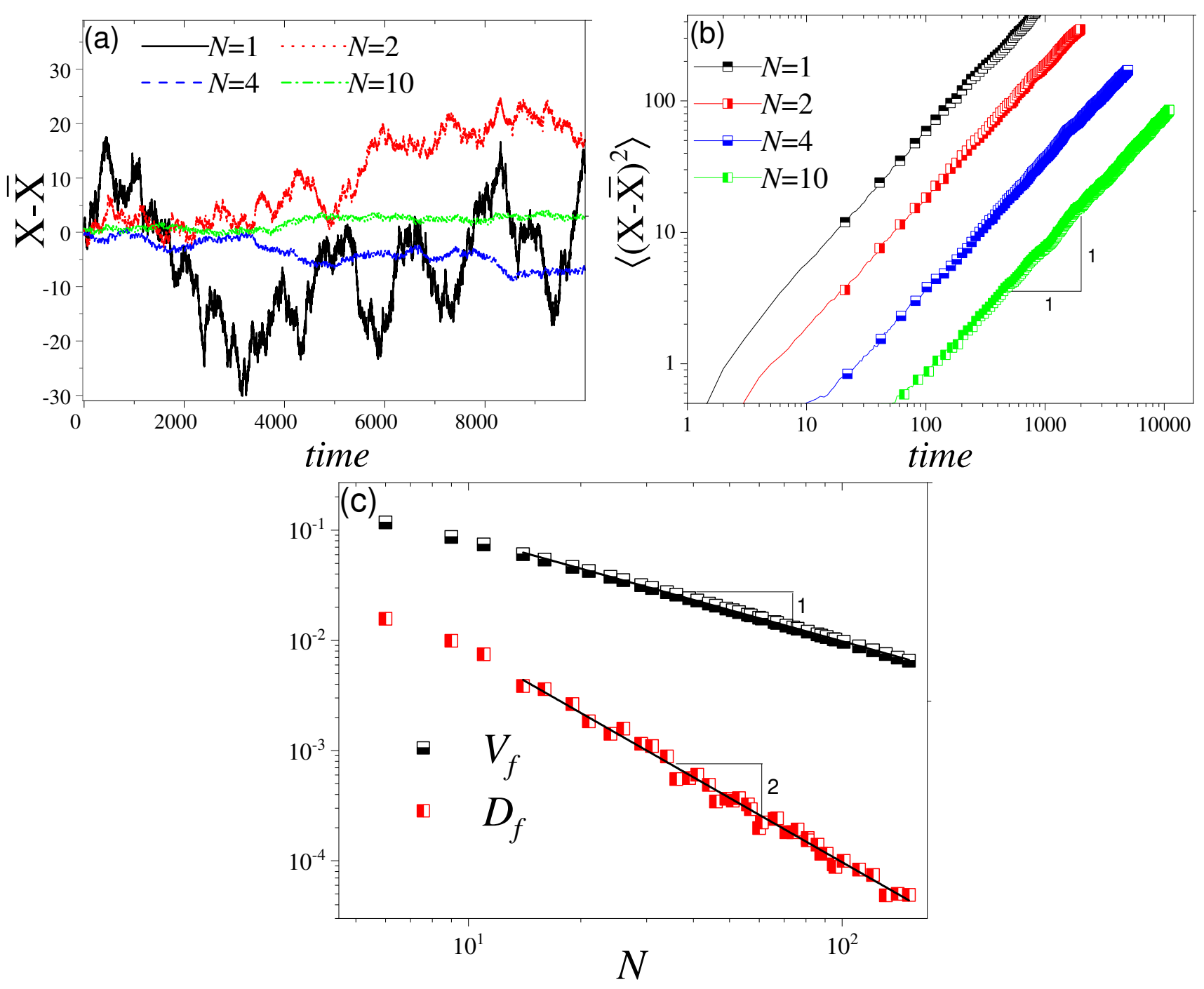

Figure 2. (a) Realization of $X-\bar{X}$ versus time for different values of $N$ for the single-species model. (b) $\left\langle(X-\bar{X})^{2}\right\rangle$ versus time for different values of $N$. The linear behavior in $\log / \log$ diagram and the slope of one ensures the random walk like behavior of fluctuations and thus we can write: $\left\langle(X-\bar{X})^{2}\right\rangle=D_{f} t$. (c) Invasion front velocity and diffusion constant versus environmental barrier intensity, $N$. For the large values of $N$, we have $V_{f} \propto N^{-\gamma_{V}}$ with $\gamma_{V}=1 \pm 0.05$ and $D_{f} \propto N^{-\gamma_{D}}$ with $\gamma_{D}=2 \pm 0.05$.

These results are acceptable because when the occupation of units would be $N$ times slower, the invasion velocity would be slower. Fluctuations in position then would be slower by the factor of $N$ and the corresponding diffusion constant should be proportional to $N^{-2}$.

We now add a second population which does not interfere with the first population except for degrading the barrier in the invasion front. As such, the two populations see each other only on the invasion front. We start the model with two cells located at the first unit and use the same definition for the border, but it does not matter which population has visited that unit. As mentioned, an un-visited unit would be occupiable only if $\zeta n_{1}+n_{2}>N$ or $n_{1}+\zeta n_{2}>N$. The positive values of $\zeta$ show the cooperation between entities and negative values would represent the competitive populations. We first try to see how the normalized diffusion constant, $N^{2} D_{f}$, depends on $\zeta$. As Figure3 (a) shows, the interaction changes the diffusion constant. Both competitive $(\zeta<0)$ and cooperative $(\zeta<0)$ populations have higher diffusion constant in respect to non-interactive populations $(\zeta=0)$. To see how interaction affects the system response to $N$, we study the behavior of $D_{f}$ versus $N$ for different interactions (Figure3 (b)). Interestingly, the magnitude of diffusion constant depends on interactions, but its behavior versus $N$, exhibited in value of $\gamma_{D}$, depends on interactions. As such, interaction leads to higher diffusion constant with smaller $\gamma_{D}$. 

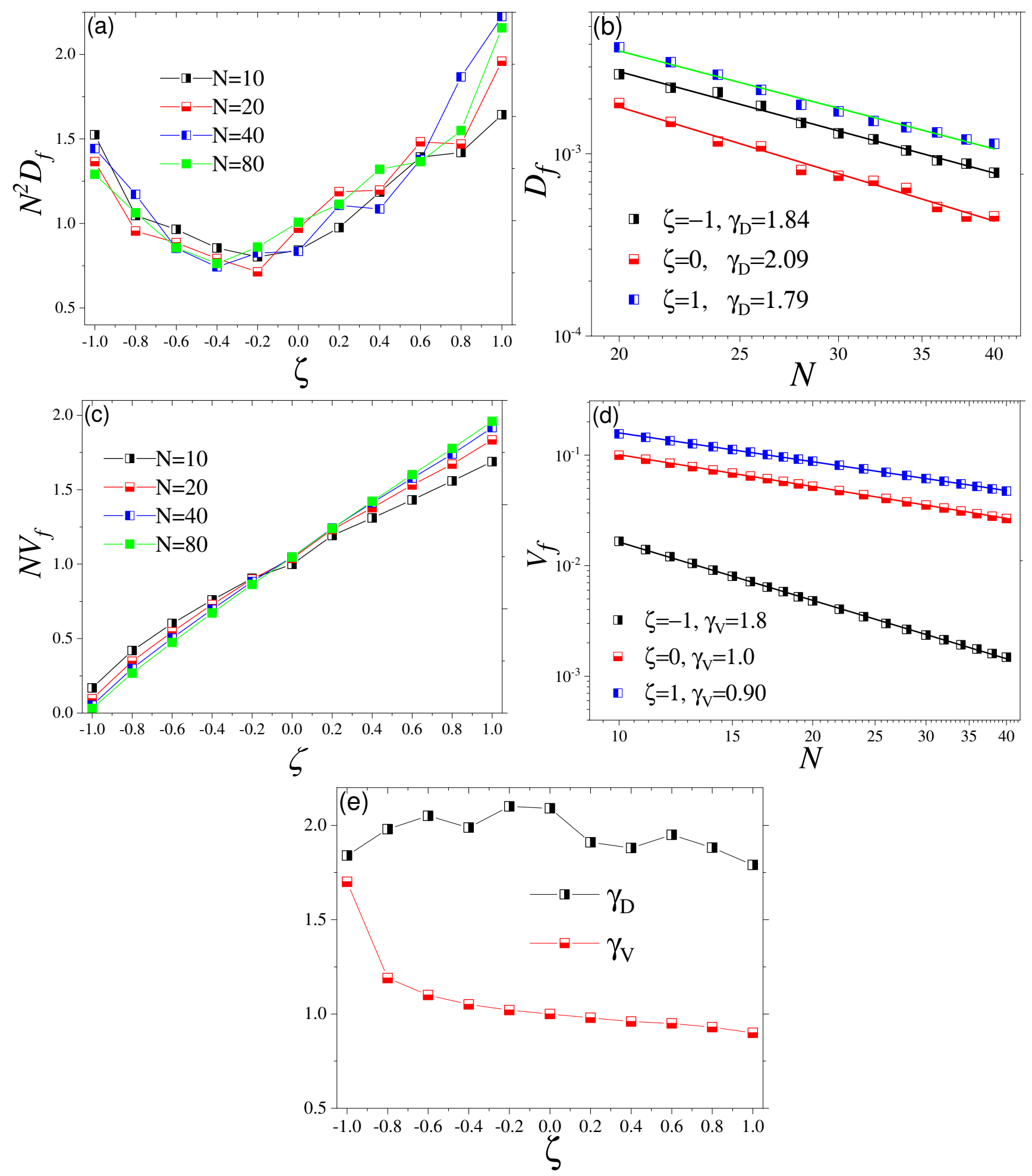

Figure 3. (a) The effect of interaction term, $\zeta$ on normalized diffusion constant, $N^{2} D_{f}$ for different values of $N$. As it shows, interaction term affects diffusion constant differently. (b) $D_{f}$ versus $N$ for different interactions. Interestingly, $\gamma_{D}$ depends on $\zeta$. (c) Normalized invasion velocity versus $\zeta$ for different values of $N$. (d) $V$ versus $N$ for different interactions. $\gamma_{V}$ also depends on $\zeta$. Based on this figure, competitive populations are more sensitive to environmental stresses. (e) $\gamma_{D}$ and $\gamma_{V}$ versus $\zeta$. While $\gamma_{V}$ monotonically decreases by $\zeta, \gamma_{D}$ has the maximum at $\zeta \sim 0$.

We also studied the effect of interactions on invasion velocity, $V_{f}$. As Figure3(c) shows, cooperation (competition) increases 
(decrease) the invasion velocity but the effect also is intensified by $N$. Analysis of behavior of $V_{f}$ versus $N$ shows that $\gamma_{V}$ also depends on $\zeta$ (Figure3(d)). Finally, as Figure 3(e) shows, $\gamma_{V}$ and $\gamma_{D}$ differently depend on interaction term, $\zeta$.

\subsection{D Case}

We consider the same rules and initial conditions for the two-dimensional case, but the environment now has the width of $L$ with periodic boundary conditions. Migration and duplication can happen into four NNs around each randomly selected unit. We study the model with both single-species and two-species cases. We study two different aspects of invasion in 2D environments: invasion velocity and the geometry of the invasion front. We consider the average location of interface, $H$, as the location of invasion front study its movements (see Figure4(a)). We set $H=\bar{X}$ in which $\bar{X}$ stands for the average value of $X$ along the border. Similar to 1D, we anticipate a Langevin equation as $d H / d t=V_{H} t+\sqrt{D_{H}} \eta(t)$ to govern the temporal evolution of $H$. We analyzed $\bar{H}$ and $H-\bar{H}$ in which $\bar{H}$ is the ensemble average. $H-\bar{H}$ fluctuates over time like a random walker and we have: $\left\langle(H-\bar{H})^{2}\right\rangle=D_{H} t$. Since $H$ is averaged over $L$ points (more accurately, $L^{D^{\prime}}$ in which $D^{\prime}$ is the fractal dimension of interface), we anticipate fluctuations of $H-\bar{H}$ to be scaled as $1 / \sqrt{L}$. As Figure4(b) shows, $D_{H} \sim L^{-\gamma_{L}}$ with $\gamma_{L} \simeq 1$ for all values of $\zeta$. Effect of $N$ on $D_{H}$ was studied and as Figure4(c) shows, $\gamma_{D}$ slightly decreases as we increase $\zeta$ and we have $\gamma_{D}=1.86 \pm 0.03$, $\gamma_{D}=1.86 \pm 0.03, \gamma_{D}=1.76 \pm 0.03$ and $\gamma_{D}=1.62 \pm 0.05$ for $\zeta=-1, \zeta=0$ and $\zeta=1$ respectively.
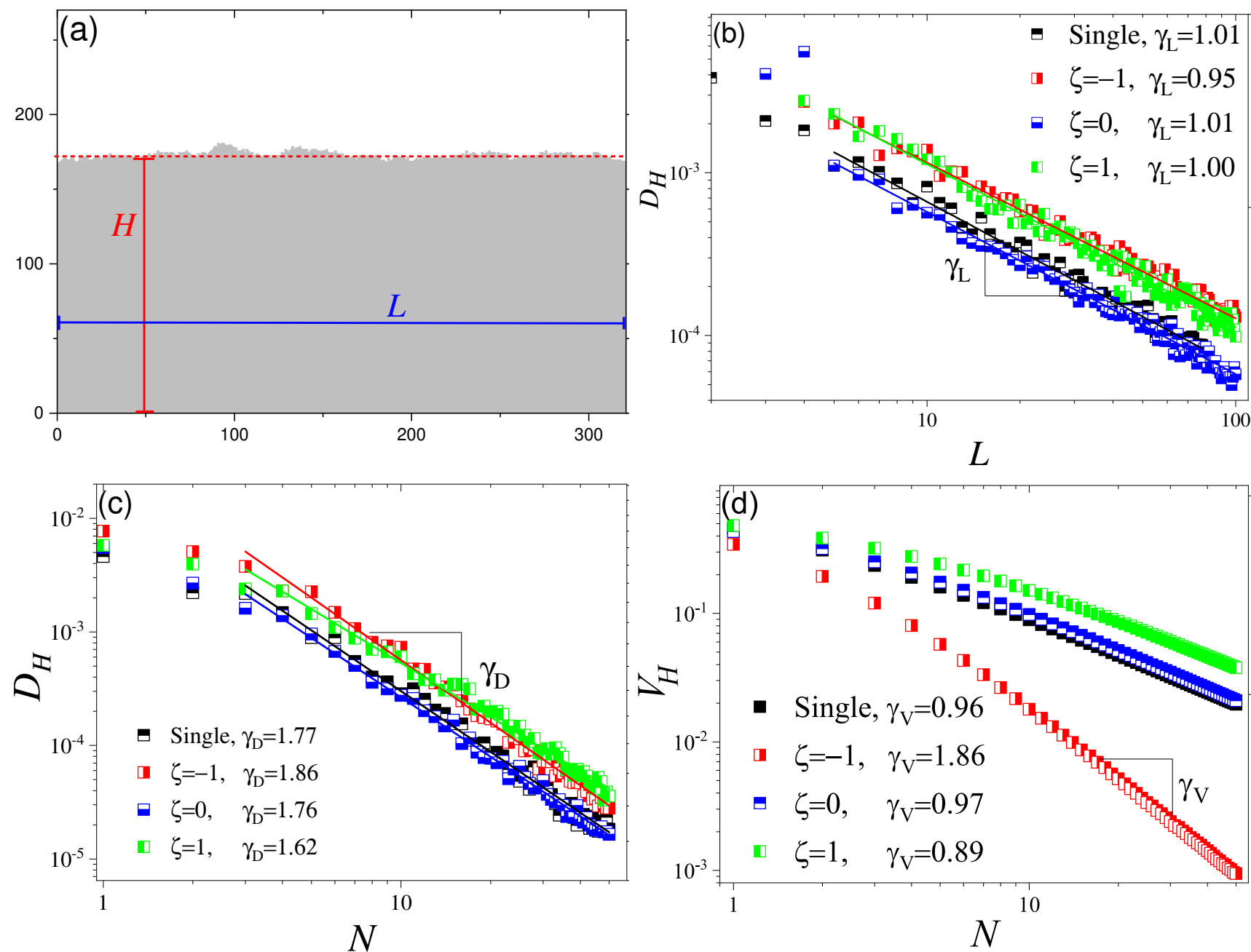

Figure 4. (a) Realization of $H-\bar{H}$ for $N=10$ and different values of $L$. (b) $D_{H}$ versus $L$ for different values of $\zeta$ compared to the single population model. As one may expect, $D_{H}$ behaves as $\sim L^{-1}$ for all interactions similarly. (c) $D_{H}$ versus $N$ for $L=20$ and different values of $\zeta$ and single population model. (d) Average velocity of interface, $V_{H}$, versus $N$.

Then we studied how $\bar{H}$ evolve during time to find the interface velocity, $V_{H}$. As Figure4(d) shows, $V_{H}$ depends on $N$ as $V_{f}$ did.

Due to importance of the geometry of invasion front, we study of the morphology of invasion front through dynamic scaling 
analysis. For such analysis, we need to calculate surface's width as $W^{2}=\frac{1}{L} \sum_{i}^{L}\left(X_{i}-H\right)^{2}$ where $X_{i}$ is the invasion front at point $i$. For variety of surfaces that follow scaling, one has $W \approx L^{\alpha} f\left(t / L^{z}\right)$ where $f(u)$ is a scaling function such that, $f(u) \propto u^{\beta}$ if $u \ll 1$, and $f(u) \approx$ constant for $u \gg 1$, so that for a fixed $L, W \propto t^{\beta}$. $\alpha$ and $\beta$ are, respectively, the surface roughness and growth exponents, and $z=\alpha / \beta$ is the dynamic exponent ${ }^{45,46}$.

We first obtain $\beta$ for different populations and as Figure5(a) shows, it decreases by $N$ for non-competitive populations. However, for competitive populations $\beta$ increases by $N$. To study the morphology of interface at steady state, we choose the saturated value of interface width, $W_{\text {sat }}$, and analyze its behavior versus $L, N$ and $\zeta$. We tried to find out whether interface follows any scaling behavior and then to see how $\zeta$ affects the corresponding exponents. As Figure 5(b) shows, $W_{\text {sat }}$ for $\zeta=-1$ is larger than other cases which indicates that invasion front of competitive populations is more rough. Later we studied the behavior of $W_{\text {sat }}$ versus $L$ and found the corresponding exponent, $\alpha_{L}$ (or simply $\alpha$ which is the roughness exponent). As Figure5(c) shows, for noncompetitive populations $(\zeta \geq 0) \alpha_{L}=0.70 \pm 0.02$ but for competitive ones $(\zeta=-1)$ we have $\alpha_{L}=0.98$. Finally, we studied the effect of $N$ on $W_{\text {sat }}$. Interestingly, as Figure5 (d) shows, competition $(\zeta=-1)$ not only leads to higher interface roughness, the associated roughness also is less sensitive to $N$. While for $\zeta=-1$ we have $\alpha_{N}=0.41$, for $\zeta \geq 0$ we have $\alpha_{N}=0.61 \pm 0.02$.
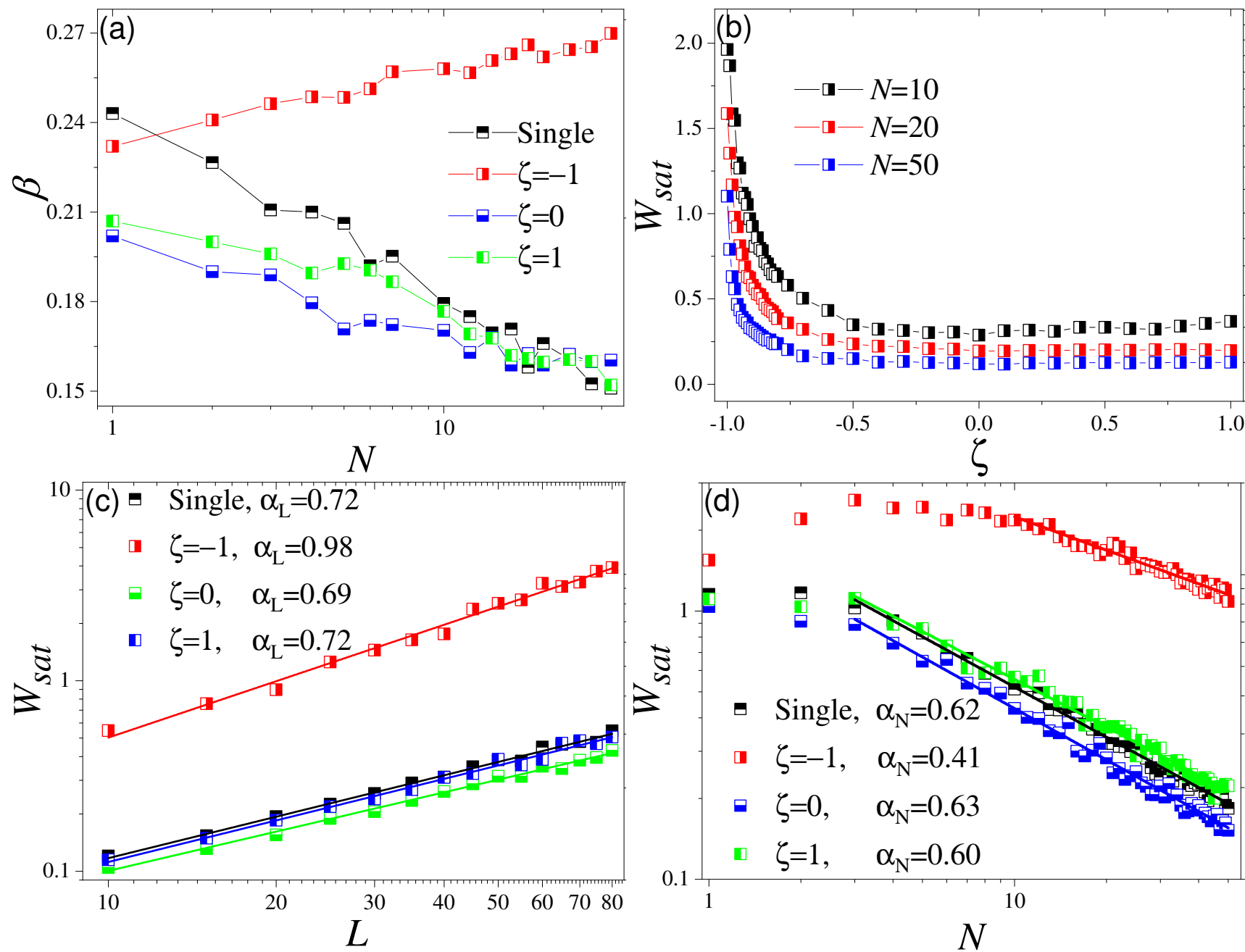

Figure 5. (a) Growth exponent, $\beta$ versus $N$ for the single population model and two interactive populations with different values for $\zeta$. As this figure shows, $N$ decreases beta only for non-competitive populations $(\zeta \geq 0)$. (b) $W_{\text {sat }}$ versus $\zeta$ for $N=10$. This figure shows that for $\zeta=-1 W_{\text {sat }}$ is much larger that other values of $\zeta$ which means that for competitive populations, invasion front might be much rough. (c) $W_{\text {sat }}$ versus $L$. The slop of $\log / \log$ diagram gives us the roughness exponent. (d) $W_{\text {sat }}$ versus $N$. For $\zeta=-1$ we have $\alpha_{N}=0.40$ and for $\zeta \geq 0$, we have $\alpha_{N}=0.61 \pm 0.02$

These results reveal that invasion front morphology and its velocity and fluctuations depend on both interspecific interactions and environmental barriers intensity. 


\section{Discussion}

Understanding invasion through mathematical modeling and in vivo or in vitro studies has significantly increased our understanding. In a now-classic example, it was suggested that duplication rate and diffusion rate of tumor cells determine tumor invasion velocity ${ }^{47}$. Since then, more parameters have been identified and taken to account to understand invasion ${ }^{48}$. Yet, a lot has been remained to be understood about how the interplay between clonal interactions and environmental stress regulate invasion.

Here we considered the physical barrier as a limiting factor that prohibits further growth and cells need to degrade it. We found how interaction significantly regulates invasion velocity. Our results suggest that cooperation plays a crucial role in cells' ability to overcome such a barrier. This conclusion is conceptually in line with other results on the relation between clonal interactions and environmental stress, such as nutrient shortage. We recently showed that once tumor cells individually acquire the ability to induce angiogenesis (angiogenic switch), they may not be able to grow larger until they cooperatively induce further angiogenesis ${ }^{49}$.

The geometry of the invasion front has been used to understand and predict tumor outcome ${ }^{33}$. As a growing interface, scaling analysis has been used to characterize the geometry of invasion front in different studies ${ }^{30,34,36}$. Most of these analyses have concentrated on how environmental features and cellular phenotype and activities such as duplication or dispersal affect the geometry of invasion front ${ }^{31,34,36}$. Here we showed that clonal interactions affect the invasion velocity and regulate the geometry of the invasion front. If we take the irregularity of invasion front as the indicator of tumor invasive behavior ${ }^{32,33}$, then we can conclude that physical barriers against growth as well as competition among different cell types can intensify invasive behavior. This conclusion is also in line with previous results on how generally competition and environmental stress together can increase invasive behavior ${ }^{43,50}$.

\section{Summary}

Motivated by clonal interactions and environmental barriers that tumor cells experience, we developed a model to study how interspecific interactions and environmental stresses together regulate invasion. In $1 \mathrm{D}$, we found the Langevin equation for invasion front and quantified the dependency of velocity and diffusion constant on the intensity of environmental barriers and the nature of interactions. It turned out that for single-species case, the invasion velocity depends on $N$ as $V_{f} \propto N^{-} \gamma_{V}$ with $\gamma_{V}=1.0$ and for the diffusion constant for invasion front, we have $D_{f} \propto N^{\gamma_{D}}$ with $\gamma_{D}=2.0$. Also, competitive populations are more vulnerable to environmental stress and their invasion velocity falls faster in response to $N$ with $\gamma_{V}=1.80 \pm 0.04$. Diffusion constant for interactive populations $(\zeta \neq 0)$ was generally larger and less sensitive to $N$ compared to non-interactive populations ( $\zeta=0$ or single population model). For the $2 \mathrm{D}$ case, the averaged invasion front $(H)$ follows a Langevin equation which depends on $N$ similar to 1D. The geometry of the invasion front exhibits scaling behavior. For $\zeta=-1$, we found that $N$ increases $\beta$. The behavior of $W_{\text {sat }}$ versus $N, L$ and $\zeta$ was obtained and it turned out that competition not only leads to more rough interfaces, but it also makes those interfaces resistant to environmental stresses. These findings deepen our understanding of the invasion of interactive species and may have applications to understanding tumor clonal interactions during the invasion.

\section{Acknowledgment}

I would like to thank Thomas Vicsek for reading the draft and his comments. I also thank the Department of Physics, the University of Tehran for computational facilities.

\section{Author contributions statement}

Y.A. designed the study, performed numerical analysis and simulations and wrote the draft.

\section{Competing financial interests}

Reprints and permissions information is available at www.nature.com/reprints. The author declares no competing financial interests.

\section{References}

1. Williamson, M. \& Griffiths, B. Biological invasions (Springer Science \& Business Media, 1996).

2. Ricciardi, A. et al. Invasion science: a horizon scan of emerging challenges and opportunities. Trends Ecol. \& Evol. 32, 464-474 (2017).

3. Van Saarloos, W. Front propagation into unstable states. Phys. Reports 386, 29-222 (2003). 
4. O'Malley, L., Korniss, G. \& Caraco, T. Ecological invasion, roughened fronts, and a competitor's extreme advance: integrating stochastic spatial-growth models. Bull. Math. Biol. 71, 1160-1188 (2009).

5. Lewis, M. A., Petrovskii, S. V. \& Potts, J. R. The mathematics behind biological invasions, vol. 44 (Springer, 2016).

6. Fisher, R. A. The wave of advance of advantageous genes. Annals Eugen. 7, 355-369 (1937).

7. Lavergne, S. \& Molofsky, J. Increased genetic variation and evolutionary potential drive the success of an invasive grass. Proc. Natl. Acad. Sci. 104, 3883-3888 (2007).

8. Korolev, K. S., Xavier, J. B. \& Gore, J. Turning ecology and evolution against cancer. Nat. Rev. Cancer 14, 371-380 (2014).

9. Wolf, K. et al. Physical limits of cell migration: control by ecm space and nuclear deformation and tuning by proteolysis and traction force. J Cell Biol 201, 1069-1084 (2013).

10. Lu, P., Takai, K., Weaver, V. M. \& Werb, Z. Extracellular matrix degradation and remodeling in development and disease. Cold Spring Harb. Perspectives Biol. 3, a005058 (2011).

11. Wirtz, D., Konstantopoulos, K. \& Searson, P. C. The physics of cancer: the role of physical interactions and mechanical forces in metastasis. Nat. Rev. Cancer 11, 512-522 (2011).

12. Spill, F., Reynolds, D. S., Kamm, R. D. \& Zaman, M. H. Impact of the physical microenvironment on tumor progression and metastasis. Curr. Opin. Biotechnol. 40, 41-48 (2016).

13. Hanahan, D. \& Weinberg, R. A. The hallmarks of cancer. Cell 100, 57-70 (2000).

14. Hanahan, D. \& Weinberg, R. A. Hallmarks of cancer: the next generation. Cell 144, 646-674 (2011).

15. Azimzade, Y. \& Saberi, A. A. Short-range migration can alter evolutionary dynamics in solid tumors. J. Stat. Mech. Theory Exp. 2019, 103502 (2019).

16. West, J., Schenck, R., Gatenbee, C., Robertson-Tessi, M. \& Anderson, A. R. Tissue structure accelerates evolution: premalignant sweeps precede neutral expansion. bioRxiv 542019 (2019).

17. Maley, C. C. et al. Classifying the evolutionary and ecological features of neoplasms. Nat. Rev. Cancer 17, 605-619 (2017).

18. Cleary, A. S., Leonard, T. L., Gestl, S. A. \& Gunther, E. J. Tumour cell heterogeneity maintained by cooperating subclones in wnt-driven mammary cancers. Nat. 508, 113-117 (2014).

19. Shahriari, K. et al. Cooperation among heterogeneous prostate cancer cells in the bone metastatic niche. Oncogene (2016).

20. Calbo, J. et al. A functional role for tumor cell heterogeneity in a mouse model of small cell lung cancer. Cancer Cell 19, 244-256 (2011).

21. Martín-Pardillos, A. et al. The role of clonal communication and heterogeneity in breast cancer. BMC Cancer 19, 1-26 (2019).

22. Kim, T.-M. et al. Subclonal genomic architectures of primary and metastatic colorectal cancer based on intratumoral genetic heterogeneity. Clin. Cancer Res. 21, 4461-4472 (2015).

23. Yachida, S. et al. Distant metastasis occurs late during the genetic evolution of pancreatic cancer. Nat. 467, 1114 (2010).

24. Campbell, P. J. et al. The patterns and dynamics of genomic instability in metastatic pancreatic cancer. Nat. 467, 1109 (2010).

25. Capp, J.-P. et al. Group phenotypic composition in cancer. Elife 10, e63518 (2021).

26. Murray, J. D. Mathematical biology i: An introduction (2003).

27. Mikhailov, A., Schimansky-Geier, L. \& Ebeling, W. Stochastic motion of the propagating front in bistable media. Phys. Lett. A 96, 453-456 (1983).

28. Hatzikirou, H., Brusch, L., Schaller, C., Simon, M. \& Deutsch, A. Prediction of traveling front behavior in a lattice-gas cellular automaton model for tumor invasion. Comput. \& Math. with Appl. 59, 2326-2339 (2010).

29. Azimzade, Y., Sasar, M. \& Maleki, I. Invasion front dynamics in disordered environments. Sci. Reports 10, 1-10 (2020).

30. Azimzade, Y., Saberi, A. A. \& Sahimi, M. Effect of heterogeneity and spatial correlations on the structure of a tumor invasion front in cellular environments. Phys. Rev. E 100, 062409 (2019).

31. Rapin, G. et al. Roughness and dynamics of proliferating cell fronts as a probe of cell-cell interactions. Sci. Reports 11, 1-9 (2021). 
32. Pérez-Beteta, J. et al. Tumor surface regularity at $\mathrm{mr}$ imaging predicts survival and response to surgery in patients with glioblastoma. Radiol. 171051 (2018).

33. Pérez-Beteta, J. et al. Morphological mri-based features provide pretreatment survival prediction in glioblastoma. Eur. Radiol. 1-10 (2018).

34. Brú, A. et al. Super-rough dynamics on tumor growth. Phys. Rev. Lett. 81, 4008 (1998).

35. Brú, A., Albertos, S., Subiza, J. L., García-Asenjo, J. L. \& Brú, I. The universal dynamics of tumor growth. Biophys. journal 85, 2948-2961 (2003).

36. Huergo, M., Pasquale, M., González, P., Bolzán, A. \& Arvia, A. Growth dynamics of cancer cell colonies and their comparison with noncancerous cells. Phys. Rev. E 85, 011918 (2012).

37. Munn, L. L. Dynamics of tissue topology during cancer invasion and metastasis. Phys. Biol. 10, 065003 (2013).

38. Dey, B., Sekhar, G. R. \& Mukhopadhyay, S. K. In vivo mimicking model for solid tumor towards hydromechanics of tissue deformation and creation of necrosis. J. Biol. Phys. 1-40 (2018).

39. Block, M., Schöll, E. \& Drasdo, D. Classifying the expansion kinetics and critical surface dynamics of growing cell populations. Phys. Rev. Lett. 99, 248101 (2007).

40. Moglia, B., Guisoni, N. \& Albano, E. V. Interfacial properties in a discrete model for tumor growth. Phys. Rev. E 87, 032713 (2013).

41. Moglia, B., Albano, E. V. \& Guisoni, N. Pinning-depinning transition in a stochastic growth model for the evolution of cell colony fronts in a disordered medium. Phys. Rev. E 94, 052139 (2016).

42. Scianna, M. \& Preziosi, L. A hybrid model describing different morphologies of tumor invasion fronts. Math. modelling natural phenomena 7, 78-104 (2012).

43. Azimzade, Y., Saberi, A. A. \& Sahimi, M. Role of the interplay between the internal and external conditions in invasive behavior of tumors. Sci. Reports 8, 5968 (2018).

44. Ben-Jacob, E. et al. Generic modelling of cooperative growth patterns in bacterial colonies. Nat. 368, 46 (1994).

45. Family, F. \& s Vicsek, T. Dynamics of fractal surfaces (World Scientific, 1991).

46. Vicsek, T. Fractal growth phenomena (World scientific, 1992).

47. Swanson, K. R., Bridge, C., Murray, J. \& Alvord Jr, E. C. Virtual and real brain tumors: using mathematical modeling to quantify glioma growth and invasion. J. neurological sciences 216, 1-10 (2003).

48. Metzcar, J., Wang, Y., Heiland, R. \& Macklin, P. A review of cell-based computational modeling in cancer biology. JCO clinical cancer informatics 2, 1-13 (2019).

49. Azimzade, Y., Saberi, A. A. \& Gatenby, R. A. Superlinear growth reveals the allee effect in tumors. Phys. Rev. E 103, 042405 (2021).

50. Anderson, A. R., Weaver, A. M., Cummings, P. T. \& Quaranta, V. Tumor morphology and phenotypic evolution driven by selective pressure from the microenvironment. Cell 127, 905-915 (2006). 